Bouke de Vries*

\title{
Should Children Have a Veto over Parental Decisions to Relocate?
}

https://doi.org/10.1515/mopp-2019-0053

Published online August 4, 2020

Abstract: Many people move house at some point during their childhood and not rarely more than once. While relocations are not always harmful for under-aged children, they can, and frequently do, cause great disruption to their lives by severing their social ties as well as any attachments that they might have to their neighbourhood, town, or wider geographical region, with long-lasting psychological effects in some cases. Since it is increasingly recognised within normative philosophy as well as within Western societies that older minors should have the final say over certain issues that significantly affect their lives (think, for instance, of custody disputes, decisions about whether to get specific vaccinations or use contraceptives), this raises the question: Can it be morally incumbent upon parents to give their minor children a veto over family relocation? This article argues that the answer is affirmative. Specifically, it suggests that such duties exist if and only if (i) parents are not morally required to either relocate their families or stay put, (ii) the stakes of the decision about a family relocation are fairly low, and (iii) the children have the competence to make these decisions, as many older minors do.

Keywords: residential mobility, relocations, parental authority, children's rights, family ethics

\section{Introduction}

A significant proportion of people relocate during their childhood and not rarely more than once. Among American children aged 1-17, for instance, about 13 percent moved in 2011 (Murphey, Bandy, and Moore 2012). Likewise, 2016 census data reveal that more than 15 percent of Australian minors moved between ages 0 5, with the level of residential mobility for each age below 18 not dropping under 10 percent (Australian Bureau of Statistics 2018). While relocations are not always harmful for minor children, they can and frequently do cause great disruption to

*Corresponding author: Bouke de Vries, Umeå Universitet, Department of Historical, Philosophical and Religious Studies, Humanisthuset, Umeå, 901 87, Sweden,

E-mail: bouke.devries@umu.se

https://orcid.org/0000-7797-0166

Ә Open Access. ( 2020 Bouke de Vries, published by De Gruyter. (co)BY the Creative Commons Attribution 4.0 International License. 
their lives by severing their social ties as well as any attachments that they might have to their neighbourhood, town, or wider geographical region, with potentially long-lasting psychological effects.

Since it is increasingly recognized in normative philosophy ${ }^{1}$ as well as in Western societies that older minors should have the final say over certain issues that significantly affect their lives (think, for instance, of custody disputes and decisions about whether to get specific vaccinations or use contraceptives), ${ }^{2}$ the fact that relocating is often costly for children raises the question: Can it be morally incumbent upon parents to give their minor children a veto over family relocation and, if so, when? My aim here is to answer this question, which to the best of my knowledge has not received any scholarly attention. After looking more closely at the harms that minor children might incur from being uprooted (Section 2), I suggest that there are cases where granting such vetoes would be inappropriate (Section 3). However, I go on to argue that, in other cases, parents should give their minor children the final say over a family relocation in order to respect the latter's developing autonomy (Section 4). Such moral duties exist when, and only when, (i) parents are not morally required to either relocate their families or stay put, (ii) the stakes of the decision about a family relocation are fairly low, and (iii) the children have the competence to make these decisions, as many older minors do.

Before justifying these claims, three comments are in order. The first is that, throughout this article, my focus will be on relocations that involve children changing schools and being prevented from continuing to see regularly any friends that they might have at their old place of residence. Such relocations normally exclude those within the same neighbourhood. They will also often exclude those where the wider community of which children are part moves along with them, as happens when children are members of a nomadic people or a travelling circus family. Although such relocations raise moral concerns as well, addressing these here would take us too far afield.

The second comment is that I will concentrate exclusively on cases where parents and their children either stay put together or move together to a new place of residence, that is, as a family. A discussion of whether it is ever morally permissible for one or both parents to relocate without their minor children if the latter wish to stay behind ${ }^{3}$ would also broaden this article's scope too much.

The third comment is that accepting that it is sometimes morally incumbent upon parents to let their minor children decide about a family relocation does not

1 See, e.g. Bou-Habib and Olsaretti (2015); Clayton (2006); and MacLeod and Archard (2002).

2 Compare Daly (2018).

3 And live independently or with another parent or with other adults to whom they may or may not be related. Still another possibility is that they will live with other children in a state-run facility. 
mean that it must be morally permissible for the state to penalise parents who flout this duty, for example, by imposing fines on them. Critics might argue that this would show insufficient regard for the parents' freedom of movement. In addition, they might argue that a system that imposed such penalties would unduly interfere with the privacy of parents and children alike as excessive information about their personal lives would need to be gathered in order to determine whether any given parent or parental couple had wrongfully denied their children a veto. ${ }^{4}$

\section{The Potential Costs of Relocating for Children}

As previously mentioned, I want to start by examining some of the main harms that minor children may, and frequently do, incur from having to move house, which will help to debunk the notion that parents have an unconditional moral right to relocate their families.

One type of harm that children might incur is that they miss the natural and/or built environment of their former place of residence. For example, those moving to a city might miss the countryside and those moving to the countryside the city. Similarly, those moving from a mountainous area might miss the mountains and those moving from a coastal area the sea. Even when the environment that children leave behind has little intrinsic value compared to their new place of residence, they will often regret being cut off from it because of the significant things that have happened there. For example, a specific town may be imbued with symbolic value for a child because it has a park where she spent a cherished birthday, a cinema in which she had her first kiss, a football stadium where she would go regularly with her father to watch their favourite team, and so on.

Another important harm - indeed for many children the main harm - stems from the disruption that relocating causes to their social relationships. Consider the following testimony:

We moved several times when I was a kid as a result of Dad's employer moving him around and yes it has a big effect. You have to make new friends, get used to a new school, and so on. Basically except in rare instances I never saw my old friends again and that was very discouraging. (Bates 2017)

4 I am indebted to an anonymous reviewer for alerting me to these objections. 
Such feelings of loss about being separated from friends, which sometimes last long after the relocation, are all too common and may be especially strong among children who do not manage to re-establish close peer relationships at their new place of residence, which is in and of itself problematic. There are various factors that might hinder the re-establishment of such relationships (Anderson et al. 2014). Apart from possible differences in dialects, languages, and cultural norms and practices (construed broadly to include differences in norms and practices between different socio-economic groups), local children may have already formed peer networks with which they are (reasonably) satisfied. Indeed, in some cases, relocated children do not simply face closed social environments but positively hostile ones.

My years in this town were hellacious. I was bullied even more than ever before. New girl in eighth grade, private school, from the big city in the small town, and I was always rebellious but this made it even more so. I got into all kinds of trouble and almost didn't make it out. (S 2019)

For children to live in such hostile social environments is not only troublesome because of the pain that exposure to recurring social cruelty causes, though this clearly matters. Such exposure has also been found to engender mental health problems such as depression that often persists into adulthood (Sigurdson et al. 2015).

Yet even when there are opportunities for children to reconnect socially, not all of them will develop rewarding peer relationships at their new place of residence. For one thing, there might be internal obstacles that prevent them from seizing these opportunities. There is evidence, for instance, that children who are introverts tend to struggle to rebuild their social networks after relocation because they find initiating social contact especially challenging (Oishi and Schimmack 2010). For another, all of the available peer relationships at their new place of residence might be ones that are detrimental to their interests. Research has found that relocated male adolescents are especially likely to find themselves in this situation and, correspondingly, to be significantly more likely to become embedded in social networks with deviant peers than their sedentary counterparts even when their family has moved to a neighbourhood with lower levels of poverty (Clampet-Lundquist et al. 2011). ${ }^{5}$ Since adolescents who end up in these networks are prone to copying the dysfunctional behaviours of their peers (Haynie and South 2005), this can have highly detrimental consequences for their own lives as well as for those of others.

5 This phenomenon has been attributed to the relative openness of these networks to new members (see Haynie and South 2005). 


\section{When Unilateral Parental Decisions to Relocate Are Justified}

The fact that minor children may, and not rarely do, suffer substantial harms from relocating makes it reasonable to doubt whether parents have an unconditional moral right to relocate their families even if they should be legally allowed to do so. What is important for present purposes is that even if such unconditional moral rights do not exist, it does not follow that parents should always grant their (older) minor children a veto over family relocation. My aim in this section is to look at three conditions under which doing so would be inappropriate, before in the next section considering cases where granting such vetoes is appropriate and indeed morally required.

\subsection{Moral Duties to Stay Put}

The first of these conditions is when parents have a moral duty to refrain from relocating their families and no countervailing moral duty to do so. Such duties might arise, for instance, when children have rewarding social networks at their current place of residence and are likely to struggle to rebuild their social networks at their parents' desired place of residence because of linguistic and/or cultural differences. Another case where it may be morally incumbent upon parents to refrain from relocating their families is when (some of) their children are autistic. Since those on the autism spectrum find it notoriously difficult to deal with changes in their surroundings and daily routines, relocating can be a real nightmare for this group (e.g. Davis and Finke 2015). In both these cases, the fact that parents have fiduciary duties to ensure that their children can live at least minimally happy and flourishing lives suggests that unless there are very strong grounds for relocating, such as the necessity of providing another child with specialised medical treatment or giving essential care to an indigent grandparent, it will be morally unjustifiable for the parents to uproot their families, regardless of whether their children agree or not.

I should add at this point that it is not just children who might incur considerable harms from a family relocation. Other individuals towards whom the parents have special moral obligations may suffer as well and, in at least some of these cases, the interests of these individuals seem sufficiently weighty to impose a moral duty upon parents to refrain from relocating their families, whether or not their children consent to relocation. For example, it would appear objectionable in many cases for them to relocate their families when this prevents them from 
providing basic and potentially life-sustaining care to a sick relative or close friend. Likewise, if for example a single parent is the only physician in a small town and it is highly unlikely a replacement would be found if the parent relocated, the potential harms of a family relocation for the community's healthcare access might be so large as to impose a moral duty upon the parent to stay put.

\subsection{Moral Duties to Relocate}

The second condition under which it would be inappropriate for parents to let their minor children decide about a family relocation is when they have a moral duty to relocate their families and no countervailing moral duty to stay put. One set of cases where such duties may exist is when children are likely to enjoy a considerably better quality of life at some other place to which their family could relocate, perhaps because moving there will allow them to escape poverty and/or violence or to receive specialized medical care that they urgently require. Unless there are exceptionally strong reasons against relocating in such cases - as there may be, for instance, when doing so prevents the parents from continuing to provide life-sustaining care to a sick relative or close friend - it seems that, given parents' fiduciary responsibilities towards their minor children, the parents in question will be duty-bound to relocate their families and do so regardless of their children's support. ${ }^{6}$

Cases where parents' moral duties to relocate their families may derive from the interests of individuals other than their children, for example, providing lifesustaining care to a sick relative or close friend who lives some distance away. A different scenario could involve a parent whose expertise as a medical researcher is desperately needed in order to develop a treatment for a particular genetic disease. Even when this person's children are not afflicted by this disease, the suffering of others may be enough to impose a moral duty on her to relocate her family to a place where she can assist other scientists already working on a treatment.

6 It might be asked, as one anonymous reviewer has done: What if parents have decisive moral reasons for relocating their families and want to do so, but are not motivated by the right reasons? Suppose, for instance, that a planned family relocation will allow a child to receive much better psychological care than she does currently, but that this is not why the child's single parent wants to relocate, which is instead to be able to attend matches of his favourite football team. While the presence of such dubious motives should affect our judgements about the moral character of such parents, my sense is that that they do not impugn upon the moral permissibility of the relocation (the same is true, mutatis mutandis, when the parents' motives for staying put are based on dubious motives). However, since the question of how our motives bear on the moral character of our actions and inactions is a controversial one, I simply focus here on cases where parents have morally acceptable reasons for wanting to move. 
I have focused so far on cases where granting family relocation vetoes to children is inappropriate because it might prevent their parents from discharging their moral duties towards their children and/or towards third parties such as other relatives, friends, and co-nationals. However, some may maintain that granting such vetoes may also be inappropriate in some cases because it undermines the parents' ability to comply with self-regarding duties. For example, insofar as continuing to live at his current place of residence makes a single parent miserable, it may be argued that in the absence of very strong reasons for staying put, the parent will owe it to himself to relocate with his family to a place where he is more likely to find happiness. Though this sounds plausible to me, as the existence of self-regarding moral duties is not uncontroversial, some will question such cases. Rather than trying to prove these sceptics wrong here, which would take us too far afield, I will simply note that insofar as these cases can and do sometimes arise, they will constitute another set of cases where parents should deny family relocation vetoes to their children.

\subsection{High Stakes}

The third condition under which it would be inappropriate for parents to let their minor children decide about a family relocation is when the stakes of these decisions are high. Consider a single parent who is currently providing basic care to an indigent grandparent, which prevents him from providing basic care to another indigent grandparent, who lives on the other side of the country and who has an equally strong claim to his care. Suppose further that providing basic care to the second grandparent would require this single parent to relocate his family and prevent him from continuing to provide basic care to the first grandparent. Even when he has moral discretion over which grandparent to look after (which may be because he has conflicting moral duties to provide care to both that leave it up to him to decide whom to care for) or because he lacks moral duties to provide care to either grandparent (which may be due to the fact that he is already providing extensive care to a severely disabled child), it would seem problematic for this single parent to let any of his minor children decide about a family relocation and, in so doing, determine which grandparent will effectively be denied basic care. The reason need not be that as an adult single parent he is cognitively, epistemically, and/or psychologically better equipped to make this kind of decision than his minor children are, though many parents with minor children will be better equipped in one or more of these respects. Neither need it be the case that the children are developmentally unprepared to make such decisions, though this will be true of a proportion of minor children, especially younger ones. Even when 
none of these conditions obtain, childhood should be a period where, to the greatest feasible extent, children are not forced, encouraged or even merely allowed to make such high-stake decisions. They should be able to live a largely carefree life in the only period of their lives where this might be possible. ${ }^{7}$

What if the stakes of the decision to relocate are high, but only for the children themselves? Suppose that an educationally ambitious 12-year-old child can either attend a secondary school in her small hometown or receive a much better education at a secondary school $60 \mathrm{~km}$ away, a decision which would require her and her single parent to relocate. Suppose further that the following conditions obtain:

- The harms of relocating for the child are high, as she would have to leave behind all of her friends as well as the rural lifestyle to which she is so deeply attached.

- The harms of relocating for the child's single parent are fairly low because the parent has lived in the would-be destination city before and still has many friends there apart from the fact that he will not need to look for a new job as he is an accomplished novelist.

- Likewise, the child's friends would incur only minor harms from her leaving, as they would still have many other friends in the town.

While the stakes of the decision to relocate might only be high for the child in this case, I take it that it remains problematic for her parent to let her make this decision even if she is willing to do so. One reason for this is that choosing between her educational aspirations on the one hand and her friends and rural lifestyle on the other is likely to cause the child considerable stress. Another reason is that there is a good chance that, however she decides, she will be plagued by feelings of doubt and possibly regret afterwards. But if this is correct, then although the child will incur significant harms even when it is her parent who decides about a relocation - as the relevant value conflict will remain - not making this decision herself is likely to spare her potentially heavy psychological burdens and, consequently, render it easier for her to have a relatively carefree childhood. $^{8}$

7 Whereas a defence of the importance of being carefree for minor children is beyond this article's scope, it should be noted that it has been defended by several scholars, including Anca Gheaus (2015) and Harry Brighouse and Adam Swift (2014); in fact, Luara Ferracioli (2020) has recently gone as far as to argue that carefreeness is a necessary condition for a good childhood.

8 What about cases where the stakes of decisions about family relocations are high for minor children but they do not realise this and consequently feel little pressure in making them? While deciding about a family relocation might not be stressful for children in such cases, I take it that they might still come to question and/or regret their decision later once they understand what was at stake. Furthermore, the fact that they do not appreciate that the stakes are high raises doubts as 


\section{When Children Should Have Vetoes over Parental Decisions to Relocate}

I started this article by looking at the substantial harms that many minor children incur from relocating and then casting doubt upon the notion that parents have an unconditional moral right to relocate their families. However, I went on to argue that even when such rights do not exist, it does not follow that minor children should always be able to veto family relocations. I suggested there are cases where parents have moral duties to either stay put with their families or to relocate. In such cases, for them to let their minor children decide about a family relocation would be inappropriate as it might prevent them from fulfilling their moral duties towards their children and/or their moral duties towards other individuals. I further suggested that even when it is morally discretionary for parents to relocate their families, letting their minor children decide about a family relocation remains unjustifiable when the stakes are high. Apart from the fact that children might be developmentally ill-prepared to make these decisions and/or be less qualified to make them than their parents, I noted that allowing them to make such decisions renders it difficult for them to enjoy one of the main goods of childhood: living a largely carefree life.

My aim in this section is to suggest that there are conditions under which it is morally permissible for parents to grant family relocation vetoes to their minor children and indeed morally required for them to do so. These conditions obtain when (i) the parents lack moral duties to either relocate their families or stay put, (ii) the stakes of the decision about a family relocation are fairly low, and (iii) the children in question have the competence to make these decisions.

Having already looked at cases where parents have moral duties either to relocate their families or stay put (see the previous section), I want to focus here on conditions (ii) and (iii). Cases where the stakes of decisions about family relocations are fairly low might include ones where the parents seek:

- a place of residence with a richer cultural scene,

- a better paid job even though they currently make enough money to live comfortably,

- a bigger house even though their current house can easily accommodate all family members as well as any expected future family members, or

- simply a change of scenery.

to whether they have the requisite competence to decide about family relocations. I will say more about the competence requirement in the final section. 
Since none of these goals is a precondition for a flourishing life, let alone a minimally decent one, it seems that in the absence of weightier reasons for moving, the parents and children in question will lack fundamental interests in relocating. To the extent that this is so and there are no weighty reasons for staying put - for example, that moving is likely to worsen any behavioural or psychological problems that the children might have or that doing so will deprive a grandparent of the family's continued care - the stakes of decisions about whether to relocate will be quite modest.

It is in such cases that I believe parents ought to give their minor children the final say over a family relocation, on condition that the latter are minimally qualified to make these decisions. Exactly when children have this competence is an issue over which there can be reasonable disagreement. However, there are good grounds for thinking that those between the ages of 12 and 17 with a normal IQ and no severe mental problems will most likely have the requisite rational and psychological faculties. This is also the period during which minor children are allowed to make certain decisions about their healthcare (e.g. about whether to get specific vaccinations) as well as decisions about whether they want to work limited hours or, in the case of custody disputes, about which parent they want to live with, and so on.

Why grant family relocation vetoes to children of this age group? The answer is that this is necessary in order to respect children's developing autonomy, whereby autonomy can be broadly understood as the ability to live more or less successfully in accordance with a conception of the good life that one independently - that is, free from manipulation - endorses. ${ }^{9}$ Such respect matters not only because being denied a say over significant aspects of one's life is often detrimental to a person's well-being, though this is important. It matters most of all because treating individuals as rational or reason-responsive beings involves showing a certain level of deference to their wishes and preferences. What this means is that even if some minimally qualified children do not exercise a veto over a family relocation wisely - they might veto a relocation when they have an all-things-considered reason not to do so, perhaps because their expected wellbeing at their parents' desired place of residence is higher than their well-being at their current place of residence; or they might refrain from using their veto when they have an all-things-considered reason to use it - there remain decisive reasons for treating their wishes as binding.

At this point, some may say that insofar as it is respect for children's developing autonomy that grounds a claim to family relocation vetoes, then insofar as children's current level of autonomy equals if not exceeds that of most adults

9 This conception of personal autonomy draws on Colburn (2010). 
(perhaps because they are especially gifted children or because they have had a rocky early childhood that has made them very mature for their age), they should be given vetoes over family relocations even when the stakes are high.

The reason why this view ought to be rejected is that it gives insufficient weight to children's interests in having a largely carefree childhood, as discussed in the previous section, which are interests that exist independently of their current level of autonomy. For if it is true that being carefree is one of the main goods of childhood, then by allowing children to make high-stakes decisions about family relocations, it will be more difficult for them to enjoy this good. Consider again the case where a family that is currently providing basic care to one grandparent can only provide basic care to another grandparent if they relocate, which would prevent them from continuing to look after the first grandparent. Even if the minor children in this family are completely autonomous, letting them decide about a family relocation remains exceedingly problematic as it means that for the rest of their childhood (as well as their subsequent life), they will have to live with the reality that their decision deprived one of their grandparents of basic care. Likewise, there are good reasons for not letting the 12-year-old girl choose between her educational aspirations on the one hand - which can only be fulfilled if she and her single parent relocate to a larger city - and her friends and rural lifestyle on the other, no matter how independent the child is. For even when there are no other individuals with high stakes in her decision (as I stipulated in this scenario), this choice will be an agonising one for her and one that, once made, might well cause her to suffer feelings of doubt if not regret during the rest of her childhood (and possibly during later stages of her life as well).

To be clear, I am not denying that whether a child's decision about relocation is justifiable or not depends on the weightiness of the stakes and the child's current level of autonomy. In order to respect their developing autonomy, there needs to be a certain degree of correspondence between the level of the stakes and their capacity for individual self-direction. All that I am claiming is that the level of responsibility that children are allowed to bear should not be the same as the level that most adults are allowed to bear, as this would make it difficult for children to enjoy one of the most important goods of childhood, namely, that of being largely carefree.

Another objection that might be raised is that my argument proves too much. ${ }^{10}$ If parents should grant family relocation vetoes to their competent minor children when the stakes are not too high, then it might be thought that they should grant them vetoes over many other parental decisions as well,

10 Thanks are due to an anonymous reviewer for raising this objection. 
including decisions about where to spend family holidays, when to have family dinners and what to have for each dinner, how to divide chores among members of the household, and so on. For even if these decisions carry high stakes in some cases, they usually do not. But, the objection continues, this is a reductio ad absurdum, as it would allow children to veto most of their parents' decisions, which is likely to severely compromise the ability of families to function.

The reason why this does not follow is that minor children can only be plausibly entitled to vetoes over parental decisions on autonomy grounds when these decisions have a significant impact upon their lives (this is true even if the stakes must not be too high lest the child's ability to have a relatively carefree childhood would be unduly constrained). To see this, it should be noted that whether we live autonomously or not is determined by whether the general shape of our lives accords with our conceptions of the good life. Since decisions about a relocation have a significant impact upon children's lives, at least in cases where relocating means that they have to change schools and that their existing peer relationships are disrupted (which is the focus of this article), and since ordinarily this is not the consequence of any of the mundane decisions just mentioned, there is no reason for assuming that just because children might be entitled to vetoes on the former, they must be entitled to vetoes on the latter as well.

I want to end with two comments. The first is that when parents have moral duties to grant family relocation vetoes to their minor children, they should make reasonable efforts to ensure that their children can form independent and wellconsidered judgements about relocation. This requires, among other things, that they give their children time to ponder the matter and that they ask for their opinions at an appropriate moment. For example, it would be inappropriate to do so straight after a family fight, when a child is feeling unwell, or when there is a sibling in the room whose presence is likely to prejudice the child's response. In addition, parents might have to tell their children that they will not get angry if their children refuse to back a family relocation, let alone punish them for it.

The second comment is that while my focus has been on minor children, there may also be cases where family relocation vetoes ought to be granted to adult children who are living with their parents, which has become increasingly common in many countries due to economic crises, housing shortages, and soaring rents. A discussion of this timely topic has to await another occasion.

Acknowledgement: I want to thank the guest editors and two anonymous reviewers for helpful comments.

Research Funding: The study has been funded by Vetenskapsrådet (Swedish Research Council) under the grant number, 2018-00679. Open access funding is provided by Umeå University. 


\section{References}

Anderson, S., T. Leventhal, S. Newman, and V. Dupéré. (2014). "Residential Mobility Among Children: A Framework for Child and Family Policy." Cityscape 16 (1): 5-36.

Australian Bureau of Statistics. (2018). Population Shift: Understanding Internal Migration in Australia, Also available at https://www.abs.gov.au/ausstats/abs@.nsf/Lookup/by\% 20Subject/2071.0 2016 Main\%20Features Population\%20Shift:\%20Understanding\% 20Internal\%20Migration\%20in\%20Australia 69 (accessed June 2, 2020).

Bates, A. (2017). “Responses - Alan Bates”, Medium, https://medium.com/@alanbates/ responses.

Bou-Habib, P., and S. Olsaretti. (2015). “Autonomy and Children's Well-Being.” in A. Bagattini and C. Macleod (eds.) The Nature of Children's Well-Being: Theory and Practice (Springer Netherlands), 15-33, https://doi.org/10.1007/978-94-017-9252-3_2.

Brighouse, H., and A. Swift. (2014). Family Values: The Ethics of Parent-Child Relationships. (New Jersey: Princeton University Press).

Clampet-Lundquist, S., J. R. Kling, K. Edin, and G. J. Duncan. (2011). "Moving Teenagers Out of High-Risk Neighborhoods: How Girls Fare Better Than Boys.” American Journal of Sociology 116 (4): 1154-89.

Clayton, M. (2006). Justice and Legitimacy in (Upbringing. Oxford: Oxford University Press). Also available at http://www.oxfordscholarship.com/view/10.1093/0199268940.001.0001/ acprof-9780199268948.

Colburn, B. (2010). Autonomy and Liberalism. (New York: Routledge).

Daly, A. (2018). “Children, Autonomy and the Courts: Beyond the Right to be Heard”, In Children, Autonomy and the Courts. (Leiden: Brill Nijhoff). Also available at https://brill.com/view/ title/35870.

Davis, J. M., and E. H. Finke. (2015). "The Experience of Military Families with Children with Autism Spectrum Disorders During Relocation and Separation”, Journal of Autism and Developmental Disorders 45 (7): 2019-34.

Ferracioli, L. (2020). “Carefreeness and Children's Wellbeing”, Journal of Applied Philosophy 37 (1): 103-17.

Gheaus, A. (2015). "The "Intrinsic Goods of Childhood" and the Just Society." In The Nature of Children's Well-Being: Theory and Practice, edited by A. Bagattini and C. Macleod. (Dordrecht: Springer Netherlands), 35-52. Also available at https://doi.org/10.1007/97894-017-9252-3.

Haynie, D. L., and S. J. South (2005). "Residential Mobility and Adolescent Violence”, Social Forces 84 (1): 361-74.

MacLeod, C. M., and D. Archard, (eds.) ((2002)). The Moral and Political Status of Children. (Oxford: OUP).

Murphey, D., T. Bandy, and K. A. Moore. (2012). Frequent residential mobility and young children's well-being', Child Trends Research Brief. Also available at https://www.childtrends.org/wpcontent/uploads/2012/01/Child_Trends-2012_02_14_RB_Mobility.pdf.

Oishi, S., and U. Schimmack. (2010). "Residential Mobility, Well-Being, and Mortality”, Journal of Personality and Social Psychology 98 (6): 980-94.

Sigurdson, J. F., A. M. Undheim, J. L. Wallander, S. Lydersen, and A. M. Sund. (2015). “The LongTerm Effects of Being Bullied or a Bully in Adolescence on Externalizing and Internalizing 
Mental Health Problems in Adulthood", Child and Adolescent Psychiatry and Mental Health 9 (42). https://doi.org/10.1186/s13034-015-0075-2.

S. (2019). "Comment on the Washington Post article :Moving as a child can change who you are as an adult”, Medium. https://medium.com/@pacificsunrisephotography/this-article-is-spoton-with-my-experience-f35654751da9. 